CERN-TH/96-327

TAUP-2389-96

hep-ph/9612202

\title{
A Prediction for the 4-Loop $\beta$ Function in QCD
}

\author{
John Ellis \\ Theoretical Physics Division, CERN, CH-1211 Geneva 23, Switzerland \\ e-mail: john.ellis@cern.ch \\ Marek Karliner \\ School of Physics and Astronomy \\ Raymond and Beverly Sackler Faculty of Exact Sciences \\ Tel-Aviv University, 69978 Tel-Aviv, Israel \\ e-mail:marek@vm.tau.ac.il \\ and \\ Mark A. Samuel \\ Department of Physics, McGill University \\ Montreal,P.Q., Canada \\ and \\ Stanford Linear Accelerator Center \\ Stanford University \\ Stanford, CA 94309, USA \\ e-mail: physmas@mvs.ucc.okstate.edu
}

\begin{abstract}
We predict that the four-loop contribution $\beta_{3}$ to the QCD $\beta$ function in the $\overline{\mathrm{MS}}$ prescription is given by $\beta_{3} \simeq 23,600(900)-6,400(200) N_{f}+350(70) N_{f}^{2}+1.5 N_{f}^{3}$, where $N_{f}$ is the number of flavours and the coefficient of $N_{f}^{3}$ is an exact result from large- $N_{f}$ expansion. In the phenomenologically-interesting case $N_{f}=3$, we estimate $\beta_{3}=(7.6 \pm 0.1) \times 10^{3}$. We discuss our estimates of the errors in these QCD predictions, basing them on the demonstrated accuracy of our method in test applications to the $O(N) \Phi^{4}$ theory, and on variations in the details of our estimation method, which goes beyond conventional Padé approximants by estimating and correcting for subasymptotic deviations from exact results.
\end{abstract}

CERN-TH-96/327

November 1996

${ }^{\dagger}$ Permanent address: Department of Physics, Oklahoma State University, Stillwater, Oklahoma 74078, USA 
A perennial problem in Quantum Field Theory is the search for calculational techniques that go beyond conventional perturbation theory. These are needed in order to control higher-order terms in what are in many cases, such as QED and QCD, asymptotic perturbation series, and to make contact with essentially nonperturbative methods such as the lattice. To be credible, any proposed technique for going beyond perturbation theory must demonstrate its ability 1) to make significant predictions with 2) reliable error estimates. One candidate technique is that of Padé approximants, which have previously met these criteria in applications to problems in condensed-matter theory and statistical mechanics [1], in particular.

We have been working to extend these successes to four-dimensional Quantum Field Theories such as QED and QCD [2,3]. However, the perturbative calculations at any given order in these gauge theories are considerably more complicated than in many lower-dimensional field theories. Thus the calculated perturbative QCD series are often much shorter than those familiar in condensed-matter and statistical-mechanical applications. This is on the one hand an opportunity, because it accentuates the need for auxiliary techniques. However, it is on the other hand a challenge for the Padé approach, which is based on the available perturbative results. Shorter series provide less input into the Padé machinery, increasing unavoidably the errors and making it difficult to cross check their magnitudes.

A welcome opportunity to test the Padé method is currently provided by the perturbative series for the renormalization-group $\beta$ function in QCD [4], which is particularly interesting for several reasons:

1) It is the most fundamental quantity in perturbative QCD, and its behaviour impinges on many of the most basic issues in this theory, such as the question whether the QCD coupling strength $\alpha_{s}$ approaches a finite value in the infrared limit [5].

2) It has already been calculated exactly to three-loop order for any number of flavours $N_{f}$ [ [4], providing as much information as is available from any perturbative QCD series to be incorporated into any attempted extrapolation.

3) This and the availability of four- and five-loop calculations of the $\beta$ function in $O(N)$ scalar field theory in four dimensions [6, 7,8], which has a similar structure, facilitate the estimation of plausible errors.

4) The calculation of the four-loop contribution $\beta_{3}$ to $\beta$ seems to be within the scope of available perturbative techniques, and we are aware of at least one project [9] that is under way to evaluate it, which means that a prediction can be checked in the foreseeable future.

We predict in this paper that

$$
\beta_{3} \simeq 23,600(900)-6,400(200) N_{f}+350(70) N_{f}^{2}+1.5 N_{f}^{3}
$$

in the $\overline{\mathrm{MS}}$ prescription, where the coefficient of the last term is an exact input calculated using large- $N_{f}$ techniques [10]. In the case $N_{f}=3$ of phenomenological 
interest, we predict $\beta_{3}=(7.6 \pm 0.1) \times 10^{3}$. The estimates of the errors in our predictions are discussed in more detail below. They are based on the demonstrated accuracy of our method in test applications to the $O(N) \Phi^{4}$ theory, and on variations in the details of our estimation method, which goes beyond conventional Padé approximants by estimating and correcting for subasymptotic deviations from exact results [11].

We now review the formalism of Padé approximants [12], introducing the notation we use in this paper. Considering a general perturbative series $P(x)=\sum_{n=0}^{\infty} S_{n} x^{n}$, the Padé approximant $P_{[N / M]}(x)$ of order $[\mathrm{N} / \mathrm{M}]$ is the ratio of polynomials $A_{N}(x)$ of order $\mathrm{N}$ and $B_{M}(x)$ of order $\mathrm{M}$, chosen so that

$$
P_{[N / M]}=\frac{A_{N}(x)}{B_{M}(x)}=P(x)+\mathcal{O}\left(x^{N+M+1}\right)
$$

One may use the $P_{[N / M]}(x)$ in either of two ways: one may interpret the coefficient $S_{N+M+1}^{\text {Padé }}$ of $x^{N+M+1}$ in a power-series expansion of $P_{[N / M]}(x)$ as the Padé Approximant Prediction (PAP) for the next perturbative coefficient $S_{N+M+1}$, or one may interpret the full expression $P_{[N / M]}(x)$ as a Padé Summation (PS) of the entire series $P(x)$ [3]. In this paper, we shall mainly be concerned with refinements of the PAP's for Quantum Field-Theoretical perturbation series, but we shall also comment at the end on an application of PS.

We have exhibited previously [2.3] mathematical conditions sufficient to guarantee that PAP's converge to $S_{N+M+1}$ as $N$ and $M$ are increased. This is in particular true for any series whose perturbative coefficients have the property that

$$
\frac{S_{n+1} S_{n-1}}{S_{n}^{2}} \simeq \begin{cases} & 1+\frac{A}{n} \\ \text { or } & \\ & 1+\frac{B}{n^{2}}\end{cases}
$$

as $n \rightarrow \infty$. The first of these conditions is satisfied by any perturbative series that is dominated by a finite set of renormalon poles, and the latter condition is satisfied by the Borel transforms of such series, guaranteeing the utility of PAP's in many applications to perturbative QCD [3].

We have, moreover, been able to derive [3] asymptotic expressions for the corresponding relative errors of PAP's:

$$
\delta_{N+M+1} \equiv \frac{S_{N+M+1}^{\text {Padé }}-S_{N+M+1}}{S_{N+M+1}} \simeq\left\{\begin{array}{c}
-\frac{M ! A^{M}}{N^{M}} \\
-\frac{M ! B(B+1) \ldots(B+M-1)}{N^{2 M}}
\end{array}\right.
$$


as $N \rightarrow \infty$, for fixed $M$. There is empirical evidence that the relative errors of PAP's are fitted even better by expressions of the form (田) with the replacements

$$
N \rightarrow N+M+a M+b
$$

where the parameters $a, b$ are to be fitted on a case-by-case basis. See $^{-}$for example the comparison between PAP's and the true values of perturbative coefficients in QCD for a large number of flavours $N_{f} \rightarrow \infty$ shown in [2] and compared with asymptotic formulae for the relative errors in [13].

These convergence theorems and the successes of the asymptotic error formulae (4) lead us to propose that one use the estimated asymptotic errors to correct PAP's systematically to the Asymptotic-Padé Approximant Predictions (APAP's):

$$
S_{N+M+1}^{A P A P}=\frac{S_{N+M+1}^{\text {Padé }}}{1+\delta_{N+M+1}}
$$

We document elsewhere [11] numerical evidence that APAP's excel even over conventional PAP's in the accuracy with which they reproduce known coefficients in QCD perturbation series, using as inputs calculations of lower-order coefficients. Our objective in this paper is to predict, with a meaningful and credible error estimate, the next unknown (four-loop) term in the perturbative series for the QCD $\beta$ function, whose exact calculation by conventional methods is currently being undertaken [9]. If our prediction turns out to be correct within the stated errors, it will provide reason to believe Padé predictions for other coefficients that are less tractable to calculate exactly.

We denote the QCD $\beta$ function by $\beta(x)=-\left(\sum_{n=0}^{\infty} \beta_{n} x^{n+1}\right)$, where $x \equiv \alpha_{s} /(4 \pi)$. The known coefficients for $N_{c}=3$ in the $\overline{\mathrm{MS}}$ prescription are [4] $\beta_{0}=11-\left(2 N_{f} / 3\right)$, $\beta_{1}=102-\left(38 N_{f} / 3\right)$ and $\beta_{2}=(2857 / 2)-(5033 / 18) N_{f}+(325 / 54) N_{f}^{2}$. It is possible to use the $[0 / 1]$ PAP to estimate $\beta_{2}$ on the basis of the exact values of $\beta_{0,1}$. This is done by fitting the numerical values of $\beta_{2}\left(N_{f}\right)$ obtained from the [0/1] PAP for $N_{f}=0, \ldots, 4$ to the polynomial form $\beta_{2}=\mathcal{A}+\mathcal{B} N_{f}+\mathcal{C} N_{f}^{2}$. Since the leading coefficient of $N_{f}$ is known at each order in loop expansion [10, we anticipate here the approach to be used in the 4-loop case and include its exact value in the fit, finding

$$
\beta_{2}=946-177 N_{f}+6.02 N_{f}^{2}
$$

Although qualitatively correct, this estimate is not very accurate, which was hardly to be expected. The use we make of the [0/1] PAP is to estimate, for each individual value of $N_{f}$, the normalization coefficient $A$ or $B$ of the asymptotic correction (4), which we then use as an input in calculating the [1/1] APAP (6).

The result we obtain clearly depends on our choice between the possible asymptotic forms in (3), which is $\beta_{n+1} \beta_{n-1} / \beta_{n}^{2} \sim 1+A / n$. In contrast to the case with

\footnotetext{
${ }^{\ddagger}$ In certain simple cases such amended formulae for the relative errors (何) turn out to be exact.
} 
many QCD observables such as $\alpha_{R} / \pi \equiv\left(R_{e^{+} e^{-}}-\sum_{f} Q_{f}^{2}\right)$ in $e^{+} e^{-} \rightarrow$ hadrons, or the anomalous dimension of the quark mass insertion, we are unaware of any proof that there is a renormalon: $\beta_{n} \sim n$ ! in the $\overline{\mathrm{MS}}$ prescription used in this paper, which would be a sufficient condition for this assumption to apply [3] 3 . More is known about the $\beta$ function in supersymmetric QCD. Indeed, it is trivial in $N=2$ QCD, being given entirely by the one-loop term [14. In the case of $N=1$ QCD with matter, it is known that in a background-field Pauli-Villars prescription [15

$$
\beta(g)=-\frac{g^{3}}{16 \pi^{2}} \frac{3 N_{c}-N_{f}+\gamma\left(g^{2}\right) N_{f}}{1-N_{c}\left(g^{2} / 8 \pi^{2}\right)}
$$

where $\gamma\left(g^{2}\right)$ is the anomalous dimension of the mass operator. If the perturbative coefficients in $\gamma\left(g^{2}\right)$ are dominated by a renormalon, then also $\beta_{n} \sim n$ !, and our asymptotic assumption applies [1. On the other hand, if one considers the limit $N_{c} g^{2}$ fixed and $N_{f} / N_{c}=3-\epsilon$ fixed, the numerator of $\beta(g)$ (8) is determined by the $\mathcal{O}\left(g^{2}\right)$ term in $\gamma$, and the $[1 / 1]$ PAP is exact!

These considerations support our assumption that the leading behaviours of the perturbative coefficients are as given in the top line of (3), leading to the corresponding expression (4) for the relative error, and hence justifying the corresponding APAP correction (6), though they by no means prove it.

As a cross check on our method, and to aid in assessing its likely errors, we first apply it to the $O(N) \Phi^{4}$ theory in 4 dimensions, whose $\beta$ function we denote by $\beta(g)=\sum_{n=0}^{\infty} \beta_{n} g^{n+2}$. These $\beta$ function coefficients are known for $n \leq 4$, and it is known that $\beta_{n} \sim n$ ! as $n \rightarrow \infty$ in this theory [16], justifying the APAP procedure with the first form of subasymptotic correction in (4). We follow the same procedure as advocated above for QCD, namely we use the known results for $\beta_{0,1}$ in $[0 / 1]$ PAP's for various values of $N$ and then apply the APAP correction (6) to the naive $[1 / 1]$ PAP I. Our results for $\beta_{3}$ in $O(N) \Phi^{4}$ theory are shown in Fig. 1(a). We see that the naive $[1 / 1]$ PAP falls considerably below the known exact result for $\beta_{3}$ in this case, which is known to be a polynomial in $N: \beta_{3}=\mathcal{A}+\mathcal{B} N+\mathcal{C} N^{2}+\mathcal{D} N^{3}$, shown as the solid line in Fig. 1(a). It is clear from this figure that the [1/1] APAP with the $A / n$ type of correction (4) is startlingly accurate, reproducing qualitatively the known polynomial form and quantitatively the values of $\beta_{3}$ for $N \leq 4$. We have in fact determined empirically that the most accurate prediction of the coefficients in the polynomial expansion of $\beta_{3}$ may be obtained by a naive average $\langle A\rangle$ of the correction coefficient $A$ over the values $N=0,1,2,3,4$ studied. Using this $\langle A\rangle$

\footnotetext{
${ }^{\S}$ Consider, however, a generic change in renormalization prescription from $\overline{\mathrm{MS}}$ to one in which the QCD correction to an observable (e.g., $\alpha_{R}$ ) is treated as an effective charge. In order to cancel the factorial growth of the $\overline{\mathrm{MS}}$ perturbative coefficients for the observable, it is clear that the $\beta$-function coefficients must also diverge factorially.

"We note that the positions of renormalon singularities are not thought to depend on the renormalization scheme chosen, just their strengths. Therefore, one may expect this conclusion to carry over to the $\overline{\mathrm{MS}}$ prescription used in this paper.

"This was used as an order-of-magnitude estimate in [17].
} 
prescription, and then making a polynomial fit to $\beta_{3}=\mathcal{A}+\mathcal{B} N+\mathcal{C} N^{2}+\mathcal{D} N^{3}$, with the exactly known value of the $\mathcal{O}\left(N^{3}\right)$ coefficient $\mathcal{D}$ [6, , ], 8 , we find the numerical values of the polynomial coefficients shown in Table I, reported together with their errors relative to the known exact coefficients **. For comparison, we also display the relative errors found if one fits the correction coefficient $A$ individually for each value of $N_{f}$, as well as the errors found making the (disfavoured) $B / n^{2}$ correction, and the errors of the naive PAP.

Table I

APAP results for the polynomial coefficients of $\beta_{3}$ in $O(N) \Phi^{4}$

\begin{tabular}{|c|c|c|c|c|c|c|}
\hline $\begin{array}{c}\text { polynomial } \\
\text { coefficient }\end{array}$ & $\begin{array}{c}\text { exact } \\
\text { calculation }\end{array}$ & $\begin{array}{c}\text { APAP } \\
\text { prediction }\end{array}$ & $\begin{array}{c}\text { rel. error } \\
\text { APAP } \\
\langle A\rangle\end{array}$ & $\begin{array}{c}\text { rel. error } \\
\text { APAP, } \\
A\left(N_{f}\right)\end{array}$ & $\begin{array}{c}\text { rel. err. } \\
\text { APAP, } \\
\langle B\rangle\end{array}$ & $\begin{array}{c}\text { rel. err. } \\
\text { PAP }\end{array}$ \\
\hline \hline $\mathcal{A}$ & -100.46 & -104.32 & $+3.84 \%$ & $+4.69 \%$ & $-17 \%$ & $-31 \%$ \\
\hline $\mathcal{B}$ & -33.28 & -34.39 & $+3.34 \%$ & $+1.38 \%$ & $-17 \%$ & $-31 \%$ \\
\hline $\mathcal{C}$ & -2.06 & -1.67 & $-18.87 \%$ & $-17.18 \%$ & $-35 \%$ & $-45 \%$ \\
\hline $\mathcal{D}$ & $6.4 \times 10^{-4}$ & input & & & & \\
\hline \hline
\end{tabular}

We note that the APAP's with the $B / n^{2}$ form of correction, also shown in Fig. 1(a), are considerably less accurate than those with the $A / n$ form, in accord with what we expect from the asymptotic results of [16]. We also note that the naive $[0 / 1]$ PAP is a significantly worse approximation to $\beta_{2}$ in $O(N) \Phi^{4}$ theory than the corresponding [0/1] PAP in QCD. This implies that the APAP correction is relatively larger in this case, and leads us to suspect that the [1/1] APAP prediction in QCD might even be more accurate than that in Fig. 1(a) or Table I.

We now make our prediction for the 4-loop $\beta$ function coefficient $\beta_{3}$ in QCD. As in the previous $O(N) \Phi^{4}$ case, we first calculate the [1/1] PAP's for $0 \leq N_{f} \leq 4$, and then make the appropriate APAP correction assuming that $\left(\beta_{n+1} \beta_{n-1}\right) / \beta_{n}^{2} \simeq$ $1+A / n$, obtaining the predictions shown as crosses in Fig. 1(b). These points are obtained by averaging the value of the asymptotic coefficient $A$ over the values of $N_{f}$ chosen, which is known to give accurate predictions in the $O(N) \Phi^{4}$ case, as we have already commented in connection with Fig. 1(a). In the particular case $N_{f}=3$ of phenomenological interest, we predict $\beta_{3}=(7.6 \pm 0.1) \times 10^{3}$, with an error which we estimate conservatively from the corresponding value of $N$ in the $\Phi^{4}$ model, and for $N_{f}=1,2,4$ we find $\beta_{3}=(17.6,12.3,3.8) \times 10^{3}$ with similar fractional errors. We also fit the APAP's to the expected polynomial dependence of form $\beta_{3}=\mathcal{A}+\mathcal{B} N_{f}+\mathcal{C} N_{f}^{2}+\mathcal{D} N_{f}^{3}$, where the $\mathcal{O}\left(N_{f}^{3}\right)$ coefficient $\mathcal{D}$ is known exactly [10], obtaining the predictions shown in Table II.

** The errors in the polynomial coefficients are correlated in the fitting procedure, and our errors for individual values of $N$ are smaller. In the particular case $N=3$, for example, we find $\beta_{3}=222.4$, to be compared with the exact result $\beta_{3}=218.8$, an error of $1.6 \%$. 
Table II

APAP results for the polynomial coefficients of $\beta_{3}$ in QCD

\begin{tabular}{|c|c|c|c|c|}
\hline coefficient & $\begin{array}{c}\text { APAP, }\langle A\rangle \\
\text { prediction }\end{array}$ & $\begin{array}{c}\text { error } \\
\text { estimate }\end{array}$ & $\begin{array}{c}\text { APAP, } A\left(N_{f}\right) \\
\text { prediction }\end{array}$ & $\begin{array}{c}\text { PAP } \\
\text { prediction }\end{array}$ \\
\hline \hline $\mathcal{A}$ & 23,557 & \pm 900 & 24,086 & 20,015 \\
\hline $\mathcal{B}$ & $-6,353$ & \pm 200 & $-6,572$ & $-5,396$ \\
\hline $\mathcal{C}$ & 346 & \pm 70 & 357 & 292 \\
\hline $\mathcal{D}$ & 1.5 (input) & - & - & - \\
\hline \hline
\end{tabular}

We believe on the basis of our $O(N) \Phi^{4}$ experience that the most reliable predictions are provided by the $\langle A\rangle$ APAP's in Fig.1(b), with the relative errors of the corresponding $O(N) \Phi^{4}$ coefficient predictions providing a conservative estimate of the errors in the QCD case. These errors, which we expect to be highly correlated as in the $\Phi^{4}$ case, are also shown in Table II, as well as the APAP's obtained using $A\left(N_{f}\right)$. We prefer the $\langle A\rangle$ APAP's, but are pleased to note that the differences are comparable with our quoted error estimates. As an exercise, we have also computed and shown in Fig. 1(b) as open circles the APAP results obtained using the $B / n^{2}$ form of correction, and also display the naive PAP's shown as diamonds. We believe that the differences from the $B / n^{2}$ APAP's are likely to be overestimates of the errors: as we have discussed above, the $B / n^{2}$ APAP's have less theoretical motivation. Finally, Table II also displays the numerical differences between our preferred APAP's and the naive PAP's.

As already mentioned, we are aware of a project underway to calculate $\beta_{3}$ exactly in $\mathrm{QCD}$, which will enable our predictions to be tested. If the exact results are consistent with the predictions of our APAP method, we will then be able to use them to predict the 5-loop coefficient $\beta_{4}$ in QCD. Fig. 2 shows the corresponding $\langle A\rangle$ and $\langle B\rangle$ predictions for $\beta_{4}$ in the $O(N) \Phi^{4}$ model, compared with the known exact result in this model $\square$. The corresponding $\langle A\rangle$ predictions for the polynomial coefficients of $\beta_{4}$ for this model are displayed in Table III. They were obtained by fitting $\langle A\rangle$ and the combination $a+b$ of subasymptotic coefficients in (5) to the known exact results for $\beta_{2}$ and $\beta_{3}$ in the $O(N) \Phi^{4}$ model. Also shown in Table III are the known exact results and the relative errors. We see in Fig. 2 that the $\langle A\rangle$ and $\langle B\rangle$ predictions are closer in this higher-order case, as one might have expected. For comparison, we recall the prediction [7] that $\beta_{4}=1405 \pm 80$, which is to be compared with the exact result $\beta_{4}=1424.29$ [8] and our prediction that $\beta_{4}=1432$,

\footnotetext{
${ }^{\dagger \dagger}$ We note in passing that our APAP approach easily revealed the sign misprint that appeared in the original version of [7, and, even after sign correction, agrees better with the corrected value [8] than with the previous value [7] that had a small numerical error. We recall that PAP's have on previous occasions shown that they can identify errors and misprints in tabulations [1].
} 
corresponding to an error of $0.56 \%$. We would hope that predictions of comparable accuracy will be possible in QCD, once the exact result for $\beta_{3}$ is available. For completeness, we also provide our APAP prediction for the 6-loop coefficient $\beta_{5}$ in the $O(N)$ model, which has not yet been computed exactly: for $N=0,1,2,3,4$, we predict $-\beta_{5}=11828,17687,24958,33802$, 44330, respectively. For comparison, we recall the prediction [7] that $-\beta_{5}=17,200 \pm 50$ in $\Phi^{4}$ theory with $N=1$.

Table III

APAP results for the polynomial coefficients of $\beta_{4}$ in $O(N) \Phi^{4}$

\begin{tabular}{|c|c|c|c|}
\hline coefficient & exact & $\begin{array}{c}\text { APAP } \\
\text { prediction }\end{array}$ & rel. error \\
\hline \hline $\mathcal{A}$ & 1002.0 & 1002.26 & $0.03 \%$ \\
\hline $\mathcal{B}$ & 385.6 & 390.61 & $1.3 \%$ \\
\hline $\mathcal{C}$ & 36.12 & 38.82 & $7.5 \%$ \\
\hline $\mathcal{D}$ & 0.576 & 0.627 & $8.8 \%$ \\
\hline $\mathcal{E}$ & -0.0013 & input & \\
\hline \hline
\end{tabular}

It is not the purpose of this paper to discuss in detail the possible phenomenological implications of our prediction for $\beta_{3}$ in QCD [18. We limit ourselves here to advertizing the fact that the conventional PS procedure, applied to our APAP result for $N_{f}$, predicts the existence of a zero in the QCD $\beta$ function, and hence an infrared fixed point in the $\overline{\mathrm{MS}}$ coupling, whose location coincides with that predicted previously by Stevenson [5] using very different arguments.

\section{Acknowledgements}

This research was supported by the Israel Science Foundation administered by the Israel Academy of Sciences and Humanities, and by a Grant from the G.I.F., the German-Israeli Foundation for Scientific Research and Development. This work was also supported by the US Department of Energy under contract No. DE-AC0376F00515 and grant No. DE-FG05-84ER40215.

\section{Note added in proof:}

After this paper was submitted, T. van Ritbergen, J.A.M. Vermaseren, and S.A. Larin presented the results of an exact calculation of the four-loop QCD $\beta$ function (hep-ph/9701390). The result in their eq. (8) contains qualitatively new color factors, corresponding to quartic Casimirs, which are not present at 1-, 2and 3-loop level, and therefore cannot be estimated using the Padé method. Consequently, our predictions should be compared with the rest of their exact ex- 
pression, as shown below. Writing $\beta_{3}=\mathcal{A}+\mathcal{B} N_{F}+\mathcal{C} N_{F}^{2}+\mathcal{D} N_{F}^{3}$, we have:

\begin{tabular}{|c|c|c|c|c|}
\hline coefficient & $\begin{array}{c}\text { APAP estimate } \\
(\text { uncertainty } \sigma)\end{array}$ & $\begin{array}{c}\text { exact } \\
\text { result }\end{array}$ & $\begin{array}{c}\text { relative } \\
\text { error }\end{array}$ & $\begin{array}{c}\text { number of } \\
\sigma \text { 's }\end{array}$ \\
\hline \hline $\mathcal{A}$ & $23,557(900)$ & 24,633 & $-4.4 \%$ & 1.20 \\
\hline $\mathcal{B}$ & $-6,353(200)$ & -6375 & $-0.35 \%$ & 0.11 \\
\hline $\mathcal{C}$ & $346(70)$ & 398.5 & $-13.2 \%$ & 0.75 \\
\hline $\mathcal{D}$ & 1.5 & 1.5 & input & - \\
\hline \hline
\end{tabular}

\section{References}

[1] M.A. Samuel, G. Li and E. Steinfelds, Phys. Rev. D48(1993)869 and Phys. Lett. B323(1994)188; M.A. Samuel and G. Li, Int. J. Th. Phys.. 33(1994)1461 and Phys. Lett. B331(1994)114.

[2] M.A. Samuel, J. Ellis and M. Karliner, Phys. Rev. Lett. 74(1995)4380.

[3] J. Ellis, E. Gardi M. Karliner and M.A. Samuel, Phys. Lett. B366(1996)268 and Phys. Rev. D54(1996)6986.

[4] O.V. Tarasov, A.A. Vladimirov, A.Yu. Zharkov, Phys.Lett. 93B(1980)429; S. Larin and J.A.M. Vermaseren, Phys. Lett. B303(1993)334, hep-ph/9302208.

[5] AC. Mattingly and P.M. Stevenson, Phys. Rev. D49(1994)437; P.M. Stevenson, Phys. Lett. B331(1994)187.

[6] A.A. Vladimirov, D.I. Kazakov and O.V. Tarasov, Sov. Phys. JETP 50(1979)521 [Zh. Eksp. Th. Fiz. 77 (1979)1035].

[7] K.G. Chetyrkin et al., Phys. Lett. 132B(1983)351.

[8] H. Kleinert et al., Phys. Lett. B272(1991)39; E - ibid. B319(1993)545 and hep-th/9503230.

[9] S. Larin and J. Vermaseren, private communications.

[10] J.A. Gracey, Phys. Lett. B373(1996)178.

[11] J. Ellis, M. Karliner and M.A. Samuel, in preparation.

[12] G.A. Baker, Jr. Essentials of Padé Approximants, Academic Press, 1975; C.M. Bender and S.A. Orszag, Advanced Mathematical Methods for Scientists and Engineers, McGraw-Hill, 1978. 
[13] J. Ellis and M. Karliner, Invited Lectures at the Int. School of Nucleon Spin Structure, Erice 1995, CERN preprint TH/95-334, hep-ph/9601280.

[14] L.V. Avdeev, O.V. Tarasov, Phys. Lett. 112B(1982)356;

[15] V. Novikov, M. Shifman, A. Vainshtein and V. Zakharov, Nucl. Phys. B229(1983)381; M.A. Shifman and A.I Vainshtein, Nucl. Phys. B277(1986)456, ibid. B359(1991)571.

[16] L.N. Lipatov, Sov. Phys. JETP 45(1977)215 [Zh.Eksp.Teor.Fiz. 72 (1977)411].

[17] A.L. Kataev and V.V. Starshenko, Mod. Phys. Lett. A10(1995)235.

[18] J. Ellis, M. Karliner, J. Reid, M.A. Samuel, E. Steinfelds, in preparation. 

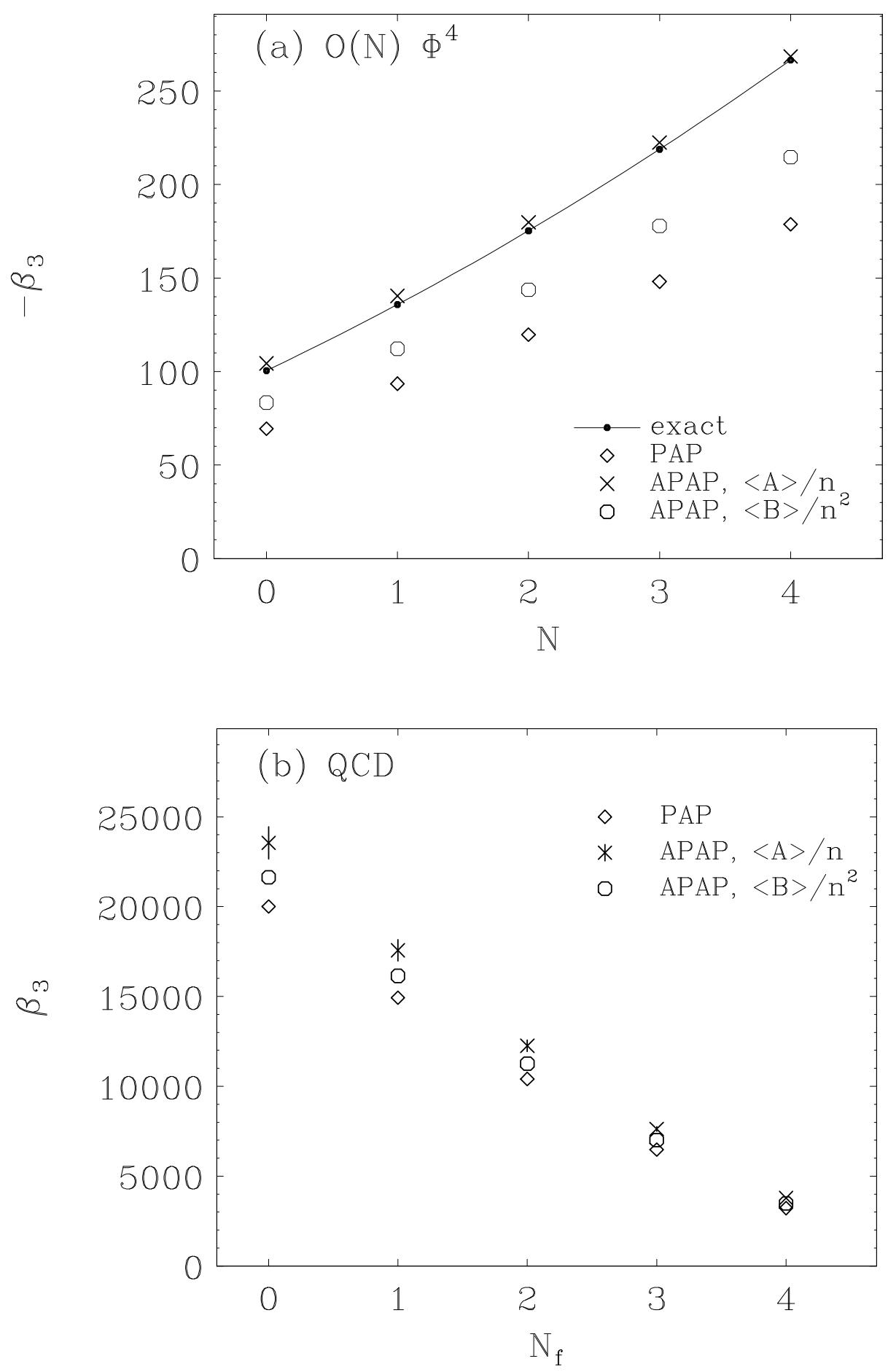

Figure 1: (a) The 4-loop $\beta$-function coefficient $\beta_{3}$ in $\Phi^{4}$ theory with $O(N)$ symmetry. The exact results are denoted by black dots, joined by a solid line to guide the eye. Naive PAP results are denoted by diamonds, and APAP results obtained from the $A / n$ type of correction are denoted by crosses. For comparison, also shown are APAP results obtained from the $B / n^{2}$ type of correction, denoted by open circles. (b) The 4-loop $\beta$-function coefficient $\beta_{3}$ in QCD with $N_{f}$ flavours, using the same notation as in Fig. 1(a). The $A / n$ APAP results are assigned error bars obtained from the relative errors in the corresponding $\Phi^{4}$ results. 


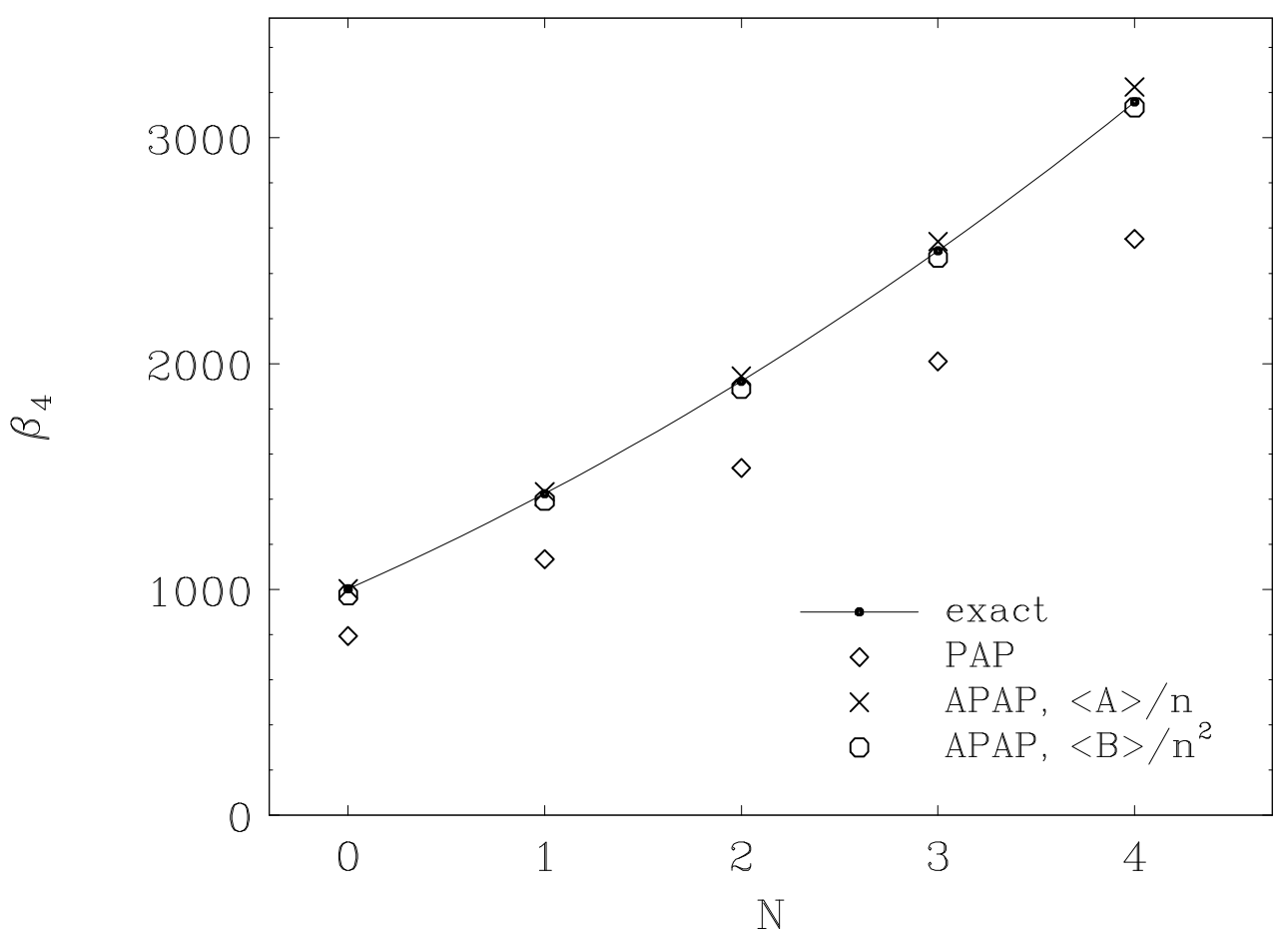

Figure 2: The 5-loop $\beta$-function coefficient $\beta_{4}$ in $\Phi^{4}$ theory with $O(N)$ symmetry, using the same notation as in Fig. 1(a). 